\title{
A Remarkable Increase in Bone Mineral Density in Celiac Disease
}

\author{
Karina Szczepanczyk ${ }^{1,2}$, Hooman Saberinia ${ }^{1}$, Faryal S. Mirza ${ }^{1,2, *}$ \\ ${ }^{1}$ Department of Medicine, UConn Health, Farmington, CT, 06030, United States of America \\ ${ }^{2}$ Division of Endocrinology and Metabolism, UConn Health, Farmington, CT, 06030, United States of America \\ *Corresponding author: fmirza@uchc.edu
}

\begin{abstract}
Introduction: Patients with active celiac disease are more likely to have osteoporosis and increased risk of fractures. Studies show that gluten-free diet may help improve bone mass to some extent. Here, we report a premenopausal woman with celiac disease with progressively declining bone mineral density (BMD) into severe osteoporosis range, who demonstrated a remarkable improvement in BMD after one year of gluten-free diet and oral bisphosphonates. Clinical case: A 44-year old premenopausal female had a low screening bone density at the ankle at 39 years of age, and was confirmed to have low BMD by central DXA, with osteoporosis at the spine and osteopenia at the hips. Celiac antibodies were positive and she was recommended gluten free diet (GFD), which she stopped after a year. Her primary care also started her on supplemental calcium and vitamin D with stabilization of BMD over the next three years. Subsequent BMD 2 years later showed $7.6 \%$ decrease in bone density at the lumbar spine (T-score -3) and 5.8\% decline at the total hip (T-score -2.7) and she was referred for osteoporosis evaluation. Workup revealed high bone turnover with an elevated PTH of $162 \mathrm{pg} / \mathrm{mL}$, low normal serum calcium of $8.8 \mathrm{mg} / \mathrm{dL}$, normal vitamin D levels and low urine calcium levels, suggestive of secondary hyperparathyroidism. At this time, review of systems revealed a questionable history of gluten sensitivity, but she denied any diarrhea or bloating. A duodenal biopsy revealed villous atrophy and positive antibodies against tissue transglutaminase, compatible with active celiac disease. She was started on a gluten-free diet and alendronate was also added in view of low bone density and high bone resorption. Bone density performed one year later revealed a remarkable $14.1 \%$ increase at the level of the lumbar spine and $8.9 \%$ increase at the level of total hip compared to prior BMD. Conclusion: This is the first case of such a remarkable increase in BMD on gluten-free diet and concomitant bisphosphonate use, suggesting a cumulative benefit of this strategy that is greater than using either of these interventions alone.
\end{abstract}

Keywords: celiac disease, osteoporosis, bone density, gluten free diet, bisphosphonate

Cite This Article: Karina Szczepanczyk, Hooman Saberinia, and Faryal S. Mirza, "A Remarkable Increase in Bone Mineral Density in Celiac Disease." International Journal of Celiac Disease, vol. 5, no. 2 (2017): 77-80. doi: 10.12691/ijcd-5-2-10.

\section{Introduction}

Celiac disease (CD) is an autoimmune disorder that affects $1 \%$ of the population worldwide. In CD, a delayed hypersensitivity reaction to gluten causes mucosal inflammation and villous atrophy of the small intestinal mucosa with malabsorption of nutrients. The combination of inflammation and malabsorption manifests as the typical gastrointestinal symptoms of diarrhea and abdominal pain. A number of extra-intestinal manifestations of this disease have also been identified, including osteopenia, anemia, neurological symptoms, infertility, dermatitis herpetiformis, and menstrual irregularities[1].

$\mathrm{CD}$ has been associated with low bone density and an increase fracture risk [2]. Inflammation, calcium and vitamin D malabsorption, sub-optimal calcium intake and secondary hyperparathyroidism have all been shown to contribute to low bone density [3]. Bone loss defects are present in $>50 \%$ of untreated celiac disease patients [4].

To date, the only treatment for $\mathrm{CD}$ is gluten free diet (GFD). Although gluten restriction can significantly reverse the systemic and local physiological mechanisms of bone deterioration in $\mathrm{CD}$ patients and improve bone mass, bone density does not restore to normal levels. Even in patients with a gluten-free diet long-term, relatively high prevalence of osteoporosis and low bone mass has been reported [5].

Less is known about the expected effects of concomitant use of GFD and bisphosphonates on bone mineral density (BMD) in this population. Here, we report a premenopausal woman with celiac disease and normal vitamin D levels with progressively declining BMD, which demonstrated a very significant increase in BMD after one year of GFD and oral bisphosphonate therapy. Although bisphosphonates are recommended in patients with osteoporosis whose BMDs persist to be in osteoporotic range on GFD, to the best of our knowledge, this is the first case reporting effect of such magnitude with this dual treatment strategy. 


\section{Case Report}

A 44-year old premenopausal female underwent screening bone density test of the ankle at 39 years of age, which showed osteopenia. Confirmatory testing with central dual-energy X-ray absorptiometry established the diagnosis of osteoporosis of the spine with lumbar spine BMD of $0.838 \mathrm{~g} / \mathrm{cm} 2, \mathrm{Z}$ score -2.4 , mean femoral neck BMD of $0.734 \mathrm{~g} / \mathrm{cm} 2, Z$ score -1.5 and mean total hip BMD of $0.711 \mathrm{~g} / \mathrm{cm} 2, Z$ score -2.0 . She did have mild symptom of bloating and diarrhea at the time. Menstrual history was unremarkable with menarche at 15 years of age. Menstrual cycles were regular. She took birth control pills from 20 to 25 years of age and again from 27 to 36 years of age. Past medical history was significant for bronchiectasis and chronic rhino-sinusitis. She did not smoke nor did she consume caffeine or alcohol. Family history was negative for CD.

Evaluation at the time by her primary care physician was positive for celiac antibodies. Total serum immunoglobulin A (IgA) was 300 units (reference range 68 -378). Gliadin peptide IgA level was 126 units (reference range $0-19,31$ or greater positive). Tissue transglutaminse (tTG) IgA antibody was 321 units (reference range 0-19). Vitamin D levels were normal at $49 \mathrm{ng} / \mathrm{ml}$. Calcium $500 \mathrm{mg}$ twice a day was added to her regimen in addition to vitamin D 1000 units daily. She was advised to start GFD at the time. She previously in 2005 had an upper endoscopy for gastroesophageal reflux disease, but duodenal biopsy was not done.
She continued supplemental calcium and vitamin D, but discontinued GFD after a year, as she did not feel much different with respect to gastrointestinal symptoms. BMD two years later was stable in spine although there was a significant decline in the hips both at the femoral neck and at total hip (Table 1, 2011). It was repeated a year later and showed improvement to baseline BMD values (Table 1, 2012). A follow up BMD 2 years later revealed significant decline at both spine and hip (Table 1, 2015), and she was referred to osteoporosis service for further evaluation. At this time she denied any diarrhea, flatulence or bloating. Physical evaluation was unremarkable without evidence of skin rash. She continued to take calcium 500 mg twice a day and vitamin D 1000 units daily in addition to a multivitamin daily during this time (2010-2015). Review of records showed persistently normal vitamin D levels in the $40-50 \mathrm{ng} / \mathrm{ml}$ range on a yearly basis from 2009 to 2015.

Laboratory evaluation revealed low serum calcium of $8.8 \mathrm{mg} / \mathrm{dl}$ (normal range 8.9-10.1 $\mathrm{mg} / \mathrm{dl}$ ) with albumin of $3.7 \mathrm{~g} / \mathrm{dl}$ (Table 2). Vitamin D was normal at $43 \mathrm{ng} / \mathrm{ml}$. Bone resorption was high as measured by urine $\mathrm{N}$ telopeptide (NTX), with an elevated parathyroid level at $162 \mathrm{pg} / \mathrm{mL}$ (normal range 10-65 pg/mL). Although she was on calcium supplements at the time, urine calcium to creatinine ratio was very low suggestive of poor calcium absorption. tTG IgA antibody was high at $>100$ (reference range 0-19). She was referred for duodenal biopsy for confirmation of CD.

Table 1. Change in bone mineral density over time

\begin{tabular}{|c|c|c|c|c|c|c|c|c|c|c|c|c|}
\hline & & & \multicolumn{3}{|c|}{ L1-L4 } & \multicolumn{3}{|c|}{ Femoral Neck } & \multicolumn{4}{|c|}{ Total Hip } \\
\hline & Age & $\begin{array}{c}\text { Weight } \\
\text { lbs }\end{array}$ & $\begin{array}{c}\text { BMD } \\
\left(\mathrm{g} / \mathrm{cm}^{2}\right)\end{array}$ & Z-Score & $\begin{array}{c}\text { \% change from } \\
\text { prior }\end{array}$ & \begin{tabular}{|c|} 
BMD \\
$\left(\mathrm{g} / \mathrm{cm}^{2}\right)$
\end{tabular} & Z-Score & $\begin{array}{l}\% \text { change } \\
\text { from prior }\end{array}$ & \begin{tabular}{|c|} 
BMD \\
$\left(\mathrm{g} / \mathrm{cm}^{2}\right)$
\end{tabular} & Z-Score & T-Score & $\begin{array}{l}\text { \% change } \\
\text { from prior }\end{array}$ \\
\hline 2009 & 39.6 & 112.4 & 0.838 & -2.4 & & 0.734 & -1.5 & & 0.711 & -2.0 & -2.8 & \\
\hline 2011 & 41.6 & 120.8 & 0.844 & -2.5 & +0.7 & 0.689 & -2.0 & -6 & 0.661 & -2.5 & -2.8 & -7 \\
\hline 2012 & 42.6 & 122.4 & 0.885 & -2.1 & +5.6 & 0.744 & -1.5 & +8 & 0.705 & -2.1 & -2.5 & +6.7 \\
\hline 2015 & 44.8 & 118.4 & 0.818 & -3.0 & -7.6 & 0.694 & -1.9 & -6.7 & 0.664 & -2.4 & -2.7 & -5.8 \\
\hline 2016 & 45.8 & 122.6 & 0.933 & -2.0 & +14 & 0.731 & -1.6 & +5.3 & 0.723 & -1.9 & -2.3 & +8.9 \\
\hline
\end{tabular}

Table 2. Laboratory evaluation over time

\begin{tabular}{|c|c|c|c|c|c|}
\hline Labs & $2 / 2015$ & $4 / 2015$ & $12 / 2015$ & $8 / 2016$ & $12 / 16$ \\
\hline Total Calcium (8.9-10.4 mg/dl) & 8.8 & & 9.3 & & 9.1 \\
\hline Albumin (3.8-5 $3 \mathrm{~g} / \mathrm{dl})$ & 3.7 & & 4.0 & & 4.2 \\
\hline Creatinine (0.6-1.2 mg/dl) & 0.7 & & 0.8 & & 0.7 \\
\hline 25 OH Vitamin D & 43 & & 83 & 57 & 48 \\
\hline Urine $\mathrm{Ca} / \mathrm{Cr}$ ratio $(\mathrm{mg} / \mathrm{g} \mathrm{Cr})$ & 7 & & 20 & & 20 \\
\hline Urine NTX (26-124 nM/Mm Cr) & Too high to calculate & 66 & 13 & & 15 \\
\hline PTH (15-88 pg/ml) & 162 & 98 & & 34 & 65 \\
\hline 1,25 OHD (19-79 pg/ml) & & 82 & & & 54 \\
\hline BS-ALP & $\begin{array}{c}23.2 \\
(7-22.4 \mathrm{ug} / \mathrm{L})\end{array}$ & & $\begin{array}{c}16.7 \\
(14.2-42.7 \mathrm{ug} / \mathrm{L})\end{array}$ & & $\begin{array}{c}15.7 \\
(14.2-42.7 \mathrm{ug} / \mathrm{L})\end{array}$ \\
\hline $\begin{array}{l}\text { TTG AB IgA } \\
<20, \text { negative } \\
>30, \text { positive }\end{array}$ & & $>100$ & & 38 & 21 \\
\hline $\begin{array}{l}\text { TTG AB IgG } \\
<20, \text { negative } \\
\geq 30, \text { positive }\end{array}$ & & 12 & & 4 & 3 \\
\hline $\mathrm{Hb}(\mathrm{g} / \mathrm{dl})$ & 14.7 & & 14.7 & & \\
\hline SPEP & Normal & & & & \\
\hline
\end{tabular}


Duodenal biopsy revealed duodenal mucosa with focal villus blunting and focal intraepithelial lymphocyte infiltrates compatible with active celiac disease. She was started on a GFD initially and alendronate was added in view of high bone resorption and significantly low BMD (she did not desire future pregnancies and her spouse had previously had vasectomy). Laboratory tests repeated at 6 and 12 months showed improvement in bone resorption and tTG IgA levels with normalization of secondary hyperparathyroidism. Urine calcium to creatinine ratio improved but still remained somewhat low (Table 2). BMD follow up one year later revealed a remarkable $14.1 \%$ increase at the level of the lumbar spine (T-score -2.1) and $8.9 \%$ increase for the total hip (T-score -2.3) (Table 1, 2016).

\section{Discussion}

Osteoporosis is a known complication of CD and should be addressed soon after diagnosis. Studies have demonstrated that $\mathrm{CD}$ patients have lower BMD and an increased risk of fractures in the peripheral skeleton. The trabecular microarchitecture is particularly impaired and may be one of the factors that diminishes bone strength and causes higher risk of fractures [6]. This emphasizes the need to recognize and treat patients early in the disease to prevent detrimental consequences. The aim of treatment in celiac patients should be to heal the underlying mucosal inflammation to allow for optimal absorption of nutrients. Unfortunately, abiding by a strict GFD is challenging for most and in many instances cannot achieve normalization of bone density, especially as an adult.

High levels of positive serology for CD have been associated with increased prevalence of osteoporosis $[5,7$, 8]. In a Canadian study using the Manitoba BMD database and provincial celiac disease serology database, 102 women were identified with the mean age of 55 years who had positive $\mathrm{CD}$ antibodies and had a bone density done at least six months prior to confirmation of celiac disease. When compared to age matched controls, subjects with celiac disease were found to have significantly lower lumbar spine, total hip and femoral neck Z scores, and a significantly higher incidence of osteoporosis on the basis of T scores. tTG antibody and IgA endomysial antibody levels correlated strongly with lower T-scores, with a significantly higher prevalence of osteoporosis in seropositive subjects at $67.7 \%$, compared to $44.4 \%$ in the seronegative subjects [8]. In a Swedish study evaluating more than 6,000 women, a higher level of tTG antibody was associated with lower bone density and a higher frequency of fractures in women 50 to 64 years of age [9].

Our patient had very high titers of celiac antibodies when her primary care physician initially checked them. Her BMD was also very low being premenopausal at the time. She had very low urine calcium suggesting poor absorption of calcium supplements and secondary hyperparathyroidism, although she had surprisingly normal vitamin D levels all along. Adherence to GFD was demonstrated by improvement in tTG IgA levels, although they are still not in the normal range. She was initiated on both GFD and alendronate in view of low BMD and high bone turnover.
Although mucosal healing is known to happen on a strict GFD, normalization of mucosal lesions and improvement of lean body mass in $\mathrm{CD}$ is a slow process and may take up to several years. In a study of 52 patients who abided by a strict GFD, Newnham et al have reported that it took 5 years for $89 \%$ of the subjects to achieve mucosal response [10]. GFD has been shown to result in significant improvement in BMD, although the magnitude of the change shown in studies is far less than was achieved with the combination treatment of GFD and alendronate in our patient. Pantaleoni et al have shown significant improvement of T-scores from -1.5 to -1.3 at the level of the femur, but not at the lumbar spine in 67 pre- and postmenopausal females after one year of a GFD [11]. In another study, Zanchetta et al showed significant improvement in BMD and bone microarchitecture as detected by high-resolution peripheral quantitative computed tomography after one year of GFD with concomitant calcium and vitamin D supplementation [6]. Overall, the gain in BMD seen in our patient at both the lumbar spine and total hip is more than the average gain reported with oral bisphosphonates over three years [12].

It is important to optimize calcium and vitamin D intake to help with improvement in bone density. The Endocrine Society clinical practice guidelines recommend a daily calcium intake of 1,000-1,500 mg daily and goal of serum vitamin D levels at $30 \mathrm{ng} / \mathrm{mL}$. Patients with celiac disease tend to have low vitamin D levels, secondary hyperparathyroidism, and high bone remodeling parameters. Interestingly, our patient was not deficient in vitamin D.

To our knowledge, this is the first reported case that demonstrates such a significant increase in BMD with the combination of GFD and bisphosphonates. The remarkable increase in BMD in our patient was likely the result of correction of several factors, including adherence to GFD with improvement in $\mathrm{CD}$, improved calcium and vitamin $\mathrm{D}$ absorption, correction of secondary hyperparathyroidism, and decreased bone resorption. Our case illustrates that bisphosphonates with GFD are an effective treatment option for osteoporosis in celiac patients, especially in those with accelerated bone loss who require rapid improvement of BMD.

\section{References}

[1] Ludvigsson, J.F., et al., The Oslo definitions for coeliac disease and related terms. Gut, 2013. 62(1): p. 43-52

[2] Hopper, A.D., et al., Adult coeliac disease. BMJ, 2007. 335(7619): p. 558-62.

[3] Tilg, H., et al., Gut, inflammation and osteoporosis: basic and clinical concepts. Gut, 2008. 57(5): p. 684-94.

[4] Corazza, G.R., et al., Bones in coeliac disease: diagnosis and treatment. Best Pract Res Clin Gastroenterol, 2005. 19(3): p. 45365.

[5] Meyer, D., et al., Osteoporosis in a north american adult population with celiac disease. Am J Gastroenterol, 2001. 96(1): p. 112-9.

[6] Zanchetta, M.B., et al., Significant bone microarchitecture impairment in premenopausal women with active celiac disease. Bone, 2015. 76: p. 149-57.

[7] Sategna-Guidetti, C., et al., The effects of 1-year gluten withdrawal on bone mass, bone metabolism and nutritional status in newly-diagnosed adult coeliac disease patients. Aliment Pharmacol Ther, 2000. 14(1): p. 35-43. 
[8] Duerksen, D.R. and W.D. Leslie, Positive celiac disease serology and reduced bone mineral density in adult women. Can J Gastroenterol, 2010. 24(2): p. 103-7.

[9] Agardh, D., et al., Coeliac disease-specific tissue transglutaminase autoantibodies are associated with osteoporosis and related fractures in middle-aged women. Scand J Gastroenterol, 2009. 44(5): p. 571-8.

[10] Newnham, E.D., et al., Adherence to the gluten-free diet can achieve the therapeutic goals in almost all patients with coeliac disease: A 5-year longitudinal study from diagnosis. J Gastroenterol Hepatol, 2016. 31(2): p. 342-9.

[11] Pantaleoni, S., et al., Bone mineral density at diagnosis of celiac disease and after 1 year of gluten-free diet. ScientificWorldJournal, 2014. 2014: p. 173082.

[12] Torring, O., Effects of denosumab on bone density, mass and strength in women with postmenopausal osteoporosis. Ther Adv Musculoskelet Dis, 2015. 7(3): p. 88-102. 